Article

\title{
Fuzzy Analytic Hierarchy Process-based Mobile Robot Path Planning
}

\author{
Changwon Kim ${ }^{1}\left(\mathbb{D}\right.$, Yeesock Kim ${ }^{2}{ }^{(D)}$, and Hak $\mathrm{Yi}^{3,+*}{ }^{3}$ \\ 1 Daegu Research Center for Medical Devices and Rehab., Korea Institute of Machinery and Materials; \\ cwkim@kimm.re.kr \\ 2 Gordon and Jill Bourns College of Engineering, California Baptist University; yekim@calbaptist.edu \\ 3 School of Mechanical Engineering, Kyungpook National University; yihak@knu.ac.kr \\ * Correspondence: yihak@knu.ac.kr; Tel.: +82-53-950-7541 \\ † Current address: School of Mechanical Engineering, Kyungpook National University, Daegu, 41566, Korea
}

\begin{abstract}
This study presents a path planning method for a mobile robot to be effectively operated through a multi-objective decision making problem. Specifically, the p roposed F uzzy analytic hierarchy process (FAHP) determines an optimal position as a sub-goal within the multi-objective boundary. The key features of the proposed FAHP is evaluating the candidates according to the fuzzified relative importance among objectives to select an optimal solution. In order to incorporate FAHP into path planning, an AHP framework is defined, which includes the highest level (goal), middle level (objectives), and the lowest level (alternatives). The distance to the target, robot's rotation, and safety against collision between obstacles are considered as objective functions. Comparative results obtained from the artificial potential field and AHP/FAHP simulations show that FAHP is much preferable for mobile robot's path planning than typical AHP.
\end{abstract}

Keywords: Fuzzy based AHP (FAHP), Multi-objective decision making, path planning, mobile robot.

\section{Introduction}

Mobile robots are requested to perform their assignment under a variety of working environments such as smart factories, highly automated hospitals, and even homes. To accomplish the given tasks, one of the important functions on mobile robots is to reach a target successfully by means of path planning. Application of many intelligence algorithms to robot's path planning is intended to implement their capability, but a lot still needs to be done [1]. Therefore this study focuses on a new way of realizing a path planning on mobile robot to generate a user's preference-based optimal path.

Path planning on mobile robots is classified into off-line and on-line methods based on the working environmental information [2,3]. Off-line path planning generates a low-resolution and high-level path due to knowledge related to a static environment, and thus provides an optimized map. Conversely, sensor technology provides on-line path planning to be robust, relative to a dynamic environment via a high-resolution low-level path [3].

Recently, there are increasing reports on an on-line path planning of a mobile robot in the dynamic environment. The early version of on-line path planning strategy was bug algorithm [4] which follows the contour of the obstacles on the way to the goal. In order to improve the inefficiency of bug algorithm, [5] suggested tangent bug algorithm assuring globally optimal path in simple environment. [6] proposed artificial potential field, that generates a mobile robot path according to the vector calculation between the goal(attractive vector) and the obstacles(repulsive vector). However, due to the 'local minima' harmonic potential filed [7] has been developed. vector field histogram (VFH) [8] generates a smooth path by observing polar histogram, while $V F H^{*}$ (the improved version of $V F H$ ) [9] predicts the consequences 
of each heading candidates. Also many of path planning research has been conducted su
rebound algorithm [10], particle swarm optimization [11], dynamic window approach [12], ect. N
on-line path planning method for efficient operation of mobile robots is still under study.

A sort of multi-objective decision making method, Analytic Hierarchy Process (AHP), is utilized to implement the proposed path planning methods. Thomas L. Saaty developed AHP back in the 1970s [13,14] that can make a decision considering several factors (objectives) simultaneously. This decision making method is widely used in a broad range of areas such as public policy, business management, industrial process scheduling, healthcare, and educational administration, etc. [15-19]. Also in engineering area, [20-22] utilized AHP method on their decision making. The application of an analytic hierarchy process (AHP) aims to implement a path planning into mobile robot platforms. The optimization process that selects optimal solutions among candidates (i.e., position in the sensing boundary) is formulated as a multi-objective optimization solution. Given the diversity of the robot working environment, it is assumed that the initial map of the space is given despite the lack of prior information on dynamic obstacles. However, AHP based decision making has a weakness that it inevitably involves decision maker's subjectivity in determining the preference for evaluation objectives. In order to address the issue of ambiguity and uncertainty resulting from the characteristic of AHP, Fuzzy based AHP (FAHP) is proposed.

By combining triangular membership function and triangular fuzzy number with AHP, Laarhoven and Pedrycz [23] developed FAHP to quantitatively include the subjectivity of the decision maker in the decision making process. Then, Chang [24] proposed the extent analysis method replacing Saaty's [17] nine-point pairwise scale to triangular fuzzy numbers. Complementing the weaknesses of AHP, FAHP has been applied in various fields. Anand et. al [25] used FAHP in the selection of robotic system in a manufacturing plant. FAHP is used in a supplier selection in a washing machine company [26], evaluation of inland inundation risk in urban area [27], unban land-use planning [28], and robot path selection problem [29].

The main contribution of this research includes proposing new on-line path planning method to plan the path of mobile robot under the decision maker's preferences, i.e. to adjust the weight between mobile robots' travel distance, robot's rotation, and safety according to the user's preference. In [20] the center of space where the robot can move was defined as the gap, and AHP was applied to the problem of selecting the gap generated at each moment. Meanwhile, in this paper, the candidates for the decision making are defined as the point where no obstacle exists in the maximum measuring distance of the Lidar. And AHP is applied to the problem of selecting the sub-target based on the relative importance matrix among objects considered for path planning. Also, [29] applied FAHP to choose the optimal path from one of the pre-planned paths, but this study differs in that it can be applied to a dynamic environments because it is a matter of planning the path of a mobile robot by applying FAHP.

This paper is organized as follows. Section 2 provides a detailed explanation of AHP. The application of AHP to mobile robot path planning and the simulations are described in Section 3 and 4, respectively. In Section 5 and 6, Fuzzy based AHP is introduced and the performance of FAHP is demonstrated through simulations. Especially, AHP and FAHP based mobile robot navigation performance is compared with a conventional method, artificial potential field based path planning in the following Section. Finally, the conclusions are discussed.

\section{Analytic Hierarchy Process}

Normally, several objectives are simultaneously considered during the decision making process. Given multiple of factors, it is difficult to choose a solution given multiple of factors because decisions are not merely determined by using a single factor. To this end, a multi-objective decision making method aids in selecting an optimal solution among alternatives by considering a decision maker's relative preference on each objective. 
In this study, Analytic Hierarchy Process (AHP), one of the famous multi-objective decision making methods, is applied. Specifically, AHP provides a comprehensive and rational framework to structure a decision making process to quantify its objectives, relate the objectives into overall goals, and evaluate alternative solutions [13]. In order to implement AHP, it is necessary to standardize the problem as hierarchies. In the AHP, the highest level of the hierarchy corresponds to the overall goal, and the lowest level corresponds to a list of alternatives (candidates). Problem initiator determines the relative importance of objectives, and all candidates are evaluated based on objectives. Subsequently, an alternative with the maximum value is selected as a solution. AHP based decision making complies with the following procedures as stated in Table 1.

Table 1. AHP decision making process.

\begin{tabular}{c} 
Problem analysis \\
Generation of the hierarchy structure \\
Relative importance of the objectives \\
Weight importance calculation \\
Evaluation of the consistency indexes for objectives \\
Evaluation of alternatives w.r.t each objective \\
\hline
\end{tabular}

The first step is to involve an analysis of the problem wherein the purpose of the problem to be solved, the objectives considered, and the alternatives suggested are defined. Thus, three main hierarchies, namely goal, objectives, and alternatives are composed as in Figure 1.

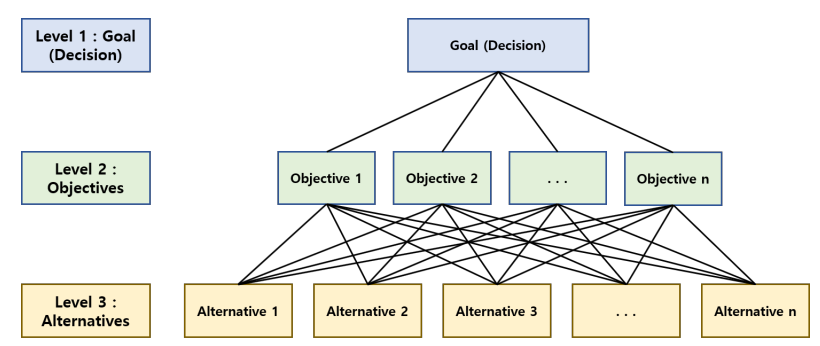

Figure 1. The structure of AHP decision making.

2

sca

95

96

Following the generation of the AHP structure, the preferences (or priority among the entries) are determined based on the importance of each objective. In the AHP framework, a pairwise comparison scale is given in accordance with a previous study [11] to define a relative importance matrix (RM). A scale is defined from 1 to 9 with the relative importance of an objective. Table 2 shows the pair-wise comparison scale in forming an $R M$.

Table 2. Pairwise comparison scale [14]

\begin{tabular}{cc}
\hline Intensity Of Importance & Definition \\
\hline 1 & Equal importance of elements \\
3 & Moderate importance of one element relative to another \\
5 & Strong importance of one element relative to another \\
7 & Very strong importance of one element relative to another \\
9 & Extreme importance of one element relative to another \\
$2,4,6,8$ & Intermediate values between two adjacent judgements \\
\hline
\end{tabular}

Specifically, $O_{n}$ represent the $n$-th objectives. When three objectives are considered, the $R M$ is formalized by using the following structure:

$$
R M=\left[\begin{array}{lll}
O_{1} / O_{1} & O_{1} / O_{2} & O_{1} / O_{3} \\
O_{2} / O_{1} & O_{2} / O_{2} & O_{2} / O_{3} \\
O_{3} / O_{1} & O_{3} / O_{2} & O_{3} / O_{3}
\end{array}\right]=\left[\begin{array}{ccc}
1 & a & b \\
1 / a & 1 & c \\
1 / b & 1 / c & 1
\end{array}\right]
$$


In equation (1), $R M$ represents a reciprocal matrix with only three elements, namely $a, b$, and $c$. To obtain a weighted objective matrix, the $R M$ is normalized according to equation (2) as follows:

$$
\mathbf{O}_{\text {norm }}=\left[\begin{array}{llll}
\frac{O_{i 1}}{\sum_{i=1}^{n} O_{i 1}} & \frac{O_{i 2}}{\sum_{i=1}^{n} O_{i 2}} & \Lambda & \frac{O_{i n}}{\sum_{i=1}^{n} O_{i n}}
\end{array}\right] .
$$

Thus, as an example, $O_{n o r m(23)}=\frac{O_{23}}{\sum_{i=1}^{n} O_{i 3}}$ where $i=1,2,3, \Lambda, n$ and $n$ denotes the number of objectives. The average of each row of equation (2) corresponds to the weighted objective matrix that is expressed as follows:

$$
\mathbf{W}_{\mathbf{o b j}}=\left[\begin{array}{llll}
\sum_{j=1}^{n} O_{\text {norm }(1, j)} & \frac{\sum_{j=1}^{n} O_{\text {norm }(2, j)}}{n} & \Lambda & \frac{\sum_{j=1}^{n} O_{\text {norm }(\kappa, j)}}{n}
\end{array},\right.
$$

where $W_{o b j}$ denotes a $1 \times n$ row vector. The weighted objective matrix shows the importance of each objective that is derived from the preference for each objective.

However, the weight objective matrix could be inappropriate [14]. Therefore, consistency of relative importance matrix should be examined because of the limitation of Saaty's discrete 9-value scale and the inconsistency of human's judgements. Saaty [13] proposed a method to measure the inconsistency. Saaty [14] proved that the largest eigenvalue of the $R M$ is equal to the size of the matrix, i.e., $\lambda_{\max }=n$, under perfect consistency. It is also possible to estimate the departure from consistency by the consistency index $(C I)$. Therefore, the $C I$ is defined as follows:

$$
C I=\frac{\lambda_{\max }-n}{n-1}
$$

After obtaining the $C I$ value, it is divided by the random consistency $(R C)$ index given in Table 3 to obtain the consistency ratio $(C R)$ as follows:

$$
C R=\frac{C I}{R C^{\prime}}
$$

Saaty [14] limits the appropriate measure as denoted by the $C R$ should not exceed 0.1 . Thus, if not so, the result is not accepted and another relative importance matrix, $R M$, is assessed until the $C R$ appropriately satisfies the condition $C R<0.1$.

Table 3. Random consistency index

\begin{tabular}{cc}
\hline number of objectives & $\boldsymbol{R C}$ \\
\hline 3 & 0.58 \\
4 & 0.90 \\
5 & 1.12 \\
6 & 1.24 \\
7 & 1.32 \\
8 & 1.41 \\
9 & 1.45 \\
\hline
\end{tabular}

After the consistency is investigated, the given alternatives are evaluated with respect to each objective. The alternative based objective evaluation matrix is given as follows:

$$
E\left(O_{i}\right)_{l m}=\frac{O_{i}\left(A_{l}\right)}{O_{i}\left(A_{m}\right)}
$$

where $O_{i}\left(A_{l}\right)$ represents the value of $l^{\text {th }}$ alternative when the $i^{\text {th }}$ objective is considered, and $i=$ $1,2, \Lambda, \gamma, m=1,2, \Lambda, \gamma$, where $\gamma$ denotes the number of alternatives. Additionally, the same process of 
121

equations (2) - (3) is used to obtain normalized matrices and the weighted alternative matrices of each corresponding objectives. The normalized form of (6) is defined as follows:

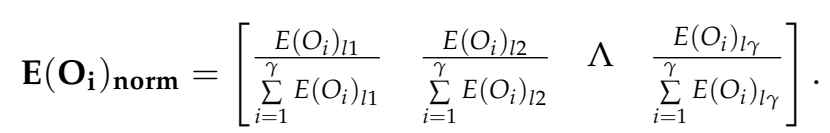

Weighted alternative matrix of each objective is obtained by using the following equation:

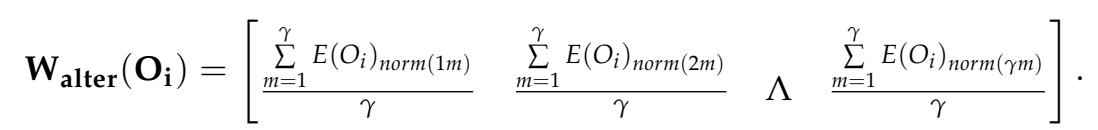

All functions are considered to obtain the following weighted alternative matrix:

$$
\mathbf{W}_{\text {alter }}=\left[\begin{array}{llll}
W_{\text {alter }}\left(O_{1}\right)^{T} & W_{\text {alter }}\left(O_{2}\right)^{T} & \Lambda & W_{\text {alter }}\left(O_{n}\right)^{T}
\end{array}\right] .
$$

A optimal function with the highest value is derived by multiplying the two resulting matrices (3) and (9) composed of weights as follows:

$$
\text { Function }^{*}=\underset{l}{\operatorname{argmax}}\left(W_{\text {alter }} \times W_{o b j}^{T}\right) .
$$

\section{AHP-based mobile robot path planning framework}

This section discusses an application of AHP to a mobile robot path planning. Prior to an analysis, it is assumed that the goal position and robot's position in global map (working space) are broadcast. Furthermore, in the mobile robot working space, a few obstacles (can be a person or objects) are randomly located such that it is not possible to specify the position. The goal involves determining an optimal waypoint within the sensor's field of view (FOV). To determine an optimal position, three objectives including distance to the target $O_{1}$, angle to the target $O_{2}$, and safety margin $O_{3}$ are considered. The alternatives for the FOV of a laser range finder (LRF) are given as follows:

$$
\begin{gathered}
P_{n}=\theta_{r}-\pi / 2+0.36 \times \pi / 180(n-1), \\
x_{P_{n}}=x_{r}+r_{s} \cos \left(P_{n}\right), \\
y_{P_{n}}=y_{r}+r_{s} \sin \left(P_{n}\right),
\end{gathered}
$$

where, the candidates are among $\pm \frac{\pi}{2}$ rad from the robot's current orientation $(n=1,2, \Lambda, 501)$. And $P_{n}$ denotes an alternatives angle, $\left(x_{p_{n}}, y_{p_{n}}\right)$ denotes an alternative position on $P_{n}, r_{s}$ represents the sensor's detection range, and the robot's current position corresponds to $\left(x_{r}, y_{r}, \theta_{r}\right)$. Figure 2 shows the coordinate system of the mobile robot. The half circle represents the field of view of LRF. The alternatives can be changed based on the sensor angle resolution (in Figure 2, only five angles are considered). 


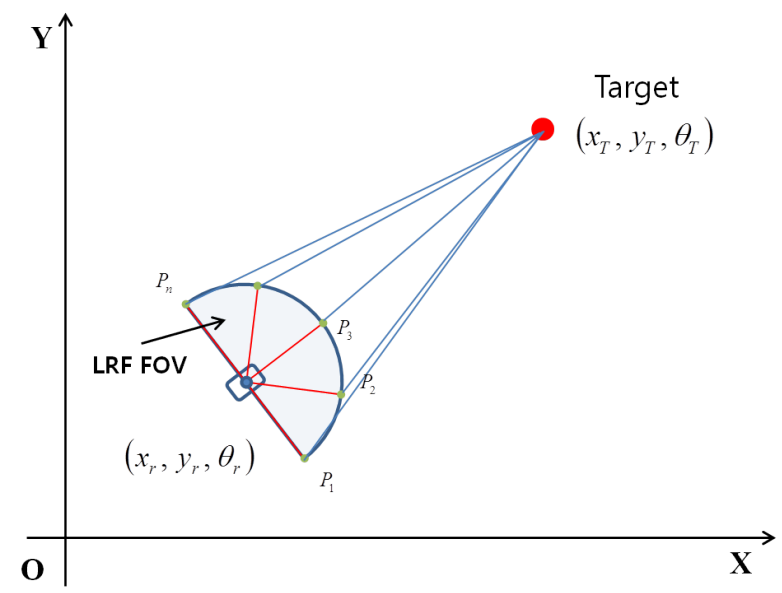

Figure 2. Coordinate system of a mobile robot with a LRF sensor.

The relative importance (i.e., $a=4, b=6, c=2$ ) is defined by user and represents that $O_{1}$ is 4 times as important as $\mathrm{O}_{2}, \mathrm{O}_{1}$ is six-times as important as $\mathrm{O}_{3}$, and $\mathrm{O}_{2}$ is twice as important as $\mathrm{O}_{3}$. Thus, the relative importance matrix among objectives is given as follows: $\mathbf{R M}_{\mathbf{1}}=\left[\begin{array}{ccc}1 & 4 & 6 \\ 1 / 4 & 1 & 2 \\ 1 / 6 & 1 / 2 & 1\end{array}\right]$. Following process is to calculate a weighted objective matrix $\left(W_{o b j}\right)$ and the consistency index of the $W_{o b j}$. Then the consistency is evaluated according to equations (4)-(5). In the given relative importance matrix, the consistency ratio $(C R=0.0079<0.1)$ satisfies an appropriate measure for the consistency. The next step involves evaluating all the candidates (alternatives, $P_{n}$ ) with respect to the objectives, namely $\mathrm{O}_{1}, \mathrm{O}_{2}$, and $\mathrm{O}_{3}$. As shown in Figure 2, different angles exhibit different distances, robot rotation angles, and safety margins. The first objective is the distance to the target, $\left(x_{t}, y_{t}, \theta_{t}\right)$, and it is expressed as follows:

$$
O_{1 p_{n}}=\sqrt{\left(x_{t}-x_{p_{n}}\right)^{2}+\left(y_{t}-y_{p_{n}}\right)^{2}} .
$$

Subsequently, the $R M$ of $O_{1}$ is given by the following expression:

$$
\mathbf{R M}\left(\mathbf{O}_{1}\right)=\left[\begin{array}{cccc}
O_{1 P_{1}} / O_{1 P_{1}} & O_{1 P_{1}} / O_{1 P_{2}} & \Lambda & O_{1 P_{1}} / O_{1 P_{n}} \\
O_{1 P_{2}} / O_{1 P_{1}} & O_{1 P_{2}} / O_{1 P_{2}} & \Lambda & O_{1 P_{2}} / O_{1 P_{n}} \\
M & M & 1 & M \\
O_{1 P_{n}} / O_{1 P_{1}} & O_{1 P_{n}} / O_{1 P_{2}} & \Lambda & O_{1 P_{n}} / O_{1 P_{n}}
\end{array}\right]
$$

The $1 \times n$ row vector weighted matrix, $W_{\text {alter }}\left(O_{1}\right)$, is obtained based on equations (2)-(3).

The second objective is the angle to the target and is expressed as follows:

$$
O_{2 P_{n}}=\tan ^{-1}\left(\frac{y_{t}-y_{p_{n}}}{x_{t}-x_{p_{n}}}\right)-\theta_{r} .
$$

The final objective corresponds to the safety margin. Figure 3 depicts the situation when the mobile robot encounters obstacles. 


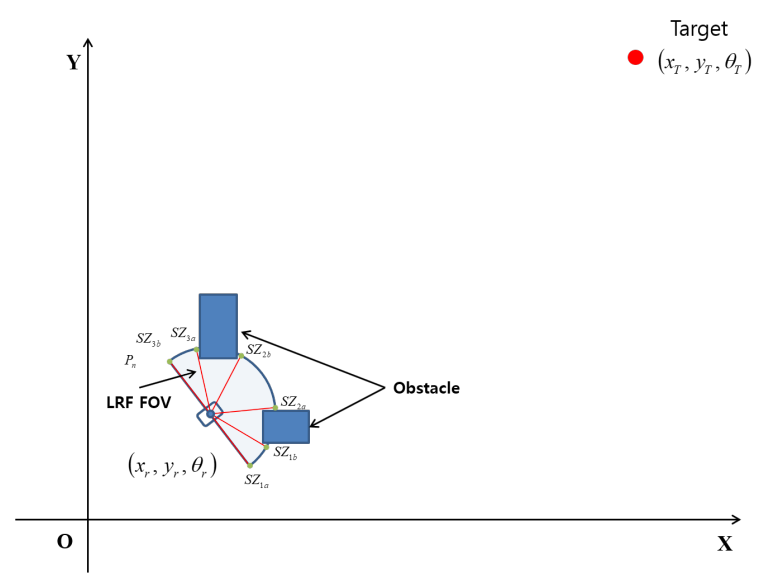

(a)

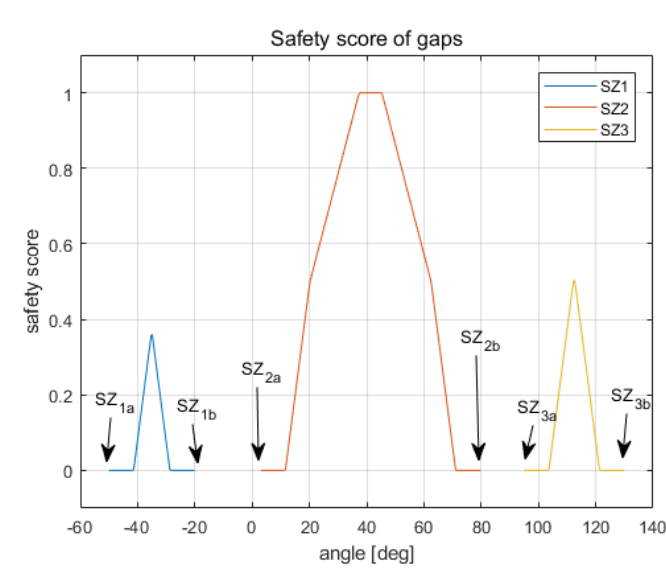

(b)

Figure 3. Safety margin under obstacles.: (a) Description of safety zone (b) Function of safety margin

Then LRF sensor provides spaces, such as $S Z_{1 a} \leq S Z_{1} \leq S Z_{1 b}, S Z_{2 a} \leq S Z_{2} \leq S Z_{2 b}$, and $S Z_{3 a} \leq S Z_{3} \leq S Z_{3 b}$, in which the robot can travel. Specifically, $S Z, S Z_{n a}$, and $S Z_{n b}$ represent the safety zone, starting angle of the $n-t h$ safety zone, and ending angle of the $n-t h$ safety zone, respectively. The basic idea of obtaining the safety margin involves utilizing the Gaussian function on two angles $S Z_{n a}$, and $S Z_{n b}$ as follows:

$$
S M\left(A_{n}\right)= \begin{cases}S M_{\text {min }}, & \left(A_{n} \leq r+r \theta_{\text {min }}\right) \\ \frac{1}{r}\left(C_{n}-r\right)+S M_{\text {min }}, & \left(r+r \theta_{\text {min }} \leq A_{n} \leq r \theta_{\text {min }}+2 r\right) \\ S M_{\text {max }}, & \left(r \theta_{\text {min }}+2 r \leq A_{n} \leq r \theta_{\text {max }}-2 r\right) \\ -\frac{1}{r}\left(C_{n}+r\right)+S M_{\text {min }}, & \left(r \theta_{\text {max }}-2 r \leq A_{n} \leq r \theta_{\text {max }}-r\right) \\ S M_{\text {min }}, & \left(A_{n} \geq r \theta_{\text {max }}-r\right)\end{cases}
$$

where $S M$ denotes the safety margin, $A_{n}=r P_{n}$ denotes arc length of $n$ - th alternative, and $\mathrm{r}$ denotes the sensing range of the robot's distance sensor. For convenience, $C_{n}=A_{n}-r \theta_{\min }$ is defined.

Given the three aforementioned objectives, all the alternatives are evaluated, and the weighted matrices for each objective are obtained as shown in equation (9) and an alternative that satisfies equation (10) is selected as the solution.

\section{Simulation I (AHP based path planning)}

In this section, the proposed path planning method is simulated on a manufacturing plant. Figure 4 shows a factory layout consisting of several work stations. The manufacturing process follows the dashed line. It is assumed that the mobile robot deliveries raw materials to each station on demand. In order to applied AHP on the path planning, the relative importance between the objectives (distance to the target, rotation of robot, and safety) should be defined. In the following simulations, two different preferences are considered, one with the highest weight on distance to the target and the other on safety. 


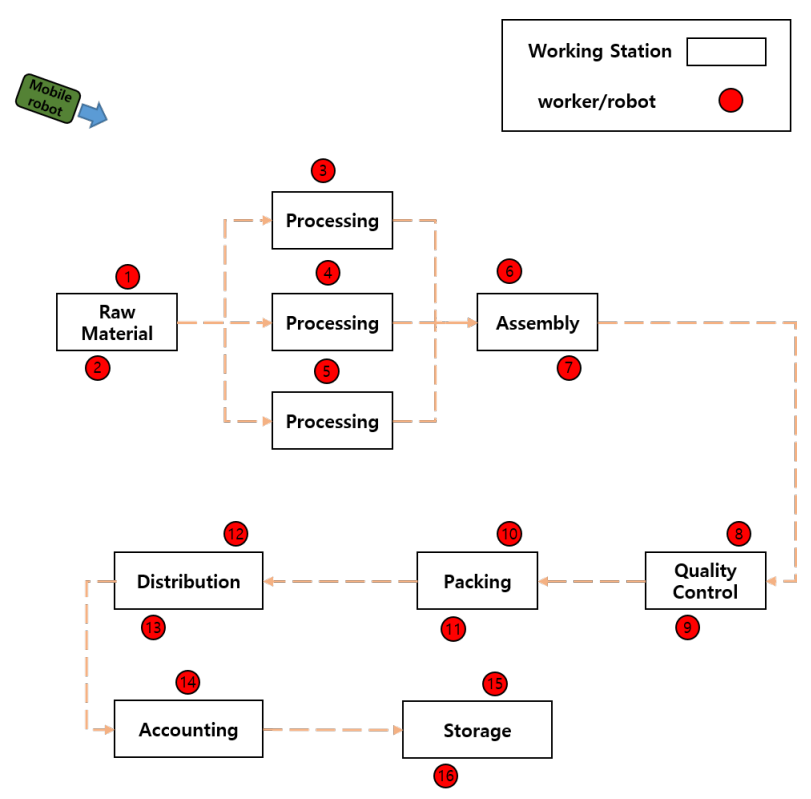

Figure 4. Factory layout.

In the manufacturing line, short traveling distance is sometimes an essential factor to improve productivity. Therefore RM is defined based on the notion that the distance to the target is four times more important than the rotation and six times the safety, and the rotation is twice as important as the safety. Therefore the RM is defined as

$$
\mathbf{R M}_{\text {dist }}=\left[\begin{array}{ccc}
1 & 4 & 6 \\
1 / 4 & 1 & 2 \\
1 / 6 & 1 / 2 & 1
\end{array}\right]
$$

The second case is designed to place safety first over other objectives. It is assumed that distance to the target and rotation are the same priority, safety is four times more important than distance to the target, and safety is four times more important than rotation. The $\mathbf{R} \mathbf{M}_{\text {safety }}$ is defined as

$$
\mathbf{R M}_{\text {safety }}=\left[\begin{array}{ccc}
1 & 1 & 1 / 4 \\
1 & 1 & 1 / 4 \\
4 & 4 & 1
\end{array}\right]
$$

The simulation is conducted utilizing equation(1)-(17). During the simulations, the total traveling distance, safety score, and number of rotation steepness are investigated. It is because the traveling distance helps to increase working efficiency, safety score ensures collision free operation, and the number of steep turn is involved in the damage to the transporting material. Figure 5 (a) shows the simulation result under $\mathbf{R} \mathbf{M}_{\text {dist }}$. while Figure 5 (b) displays the result of path planning under $\mathbf{R} \mathbf{M}_{\text {safety }}$. The $\mathbf{R} \mathbf{M}_{\text {dist }}$ based simulation results show that the total traveling distance is $81.46 \mathrm{~m}$, safety score is 50.84 , and the number of steep angles is 15 . On the other hand, the $\mathbf{R} \mathbf{M}_{\text {safety }}$ based simulation results are 84.18 of the total traveling distance, 85.30 of the safety score, and 12 times of the occurrence of steep angles. As expected, short distance traveling strategy based RM has short distance travel results, whereas safety strategy based RM has high safety scores ensuring high safety margins against collisions with obstacles. 


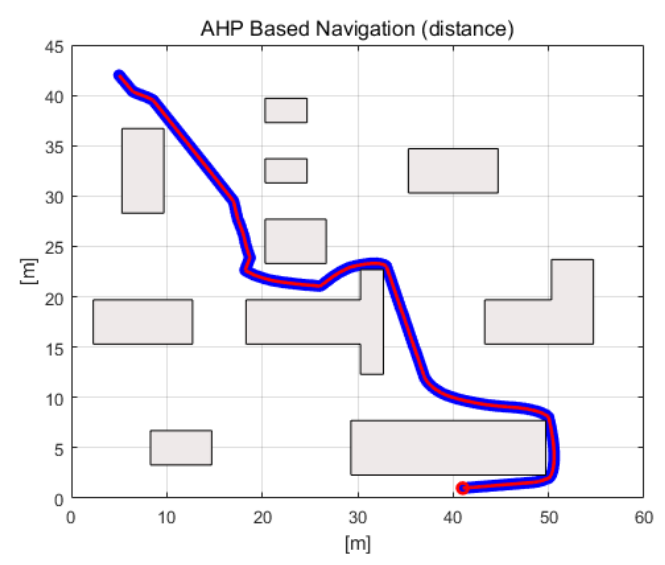

(a)

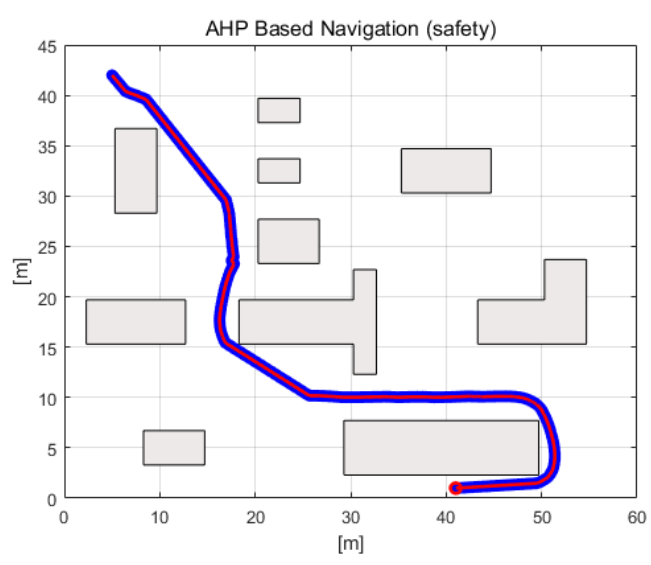

(b)

Figure 5. Safety margin under obstacles.: (a) AHP based path planning with short distance preference (b) AHP based path planning with high safety preference

Figure 6 displays the trajectory of both $R M$ s. The blue line represents $\mathbf{R M}_{\text {dist }}$ based path while the red line for $\mathbf{R M}_{\text {safety }}$. According to the defined RM, one takes shortest sub-target position while the other chooses the safest sub-target within sensing range.

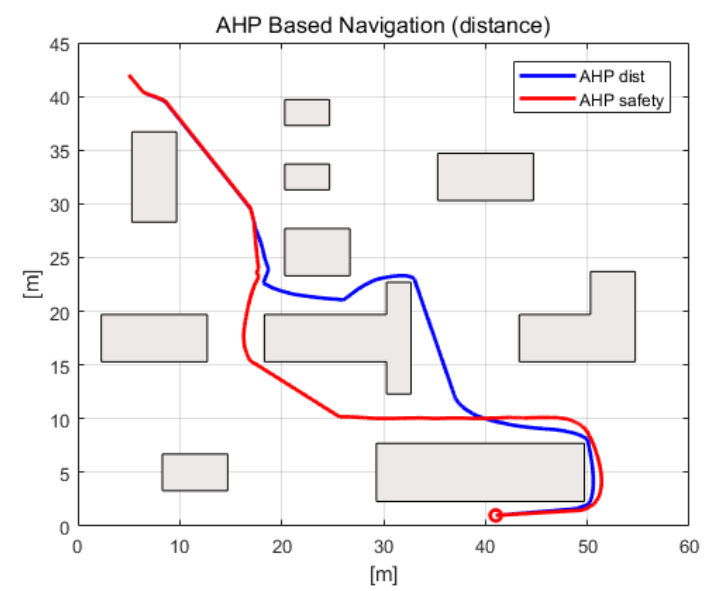

Figure 6. Comparison between short distance and high safety RM based AHP path planning.

\section{Fuzzy based AHP}

In this Section, Fuzzy based AHP (FAHP) is introduced and applied on mobile robot path planning to improve the performance of the AHP. In the framework of AHP, the pairwise comparisons is defined to select an optimal solution of given problem using Satty's nine-point scale. However, Kabir and Hasin [30] figured out the conventional AHP has the following disadvantages : (1) The AHP method creates and deals with a very unbalanced scale of judgement. (2) The AHP method does not take into account the uncertainty associated with the mapping of one's judgement to a number. (3) Ranking of the AHP method is rather imprecise. (4) The subjective judgement, selection and preference of decision makers have great influence on the AHP results. With these reasons, AHP is insufficient to explain uncertain conditions in pair-wise comparison scale. 


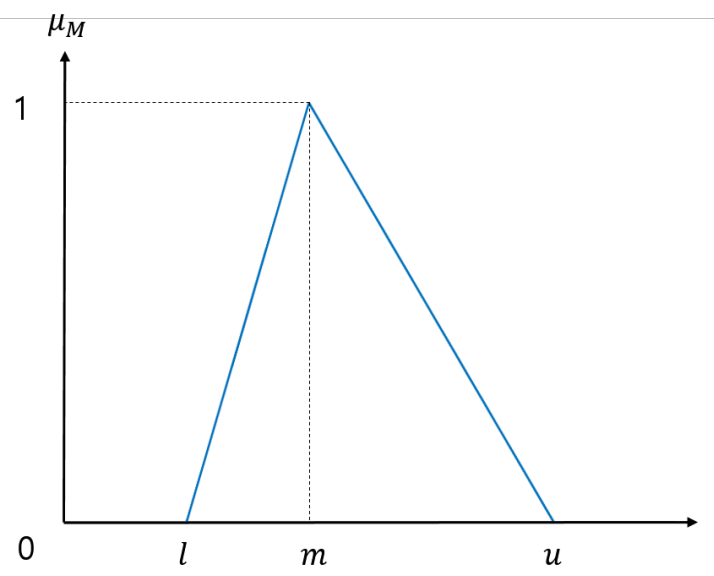

Figure 7. Fuzzy number.

In order to cover these weak-points of the AHP, FAHP is introduced. Chang [24] proposed the extent analysis method on fuzzy AHP using triangular fuzzy number as shown in Figure 8. As defined in [25], a fuzzy number $M$ on $R$ to be a triangular fuzzy number if its membership function $\mu_{M}(x)=: R \rightarrow[0,1]$ is equal to

$$
\mu_{M}(x)= \begin{cases}\frac{x}{m-l}-\frac{l}{m-l}, & \mathrm{x} \in[l, m] \\ \frac{x}{m-u}-\frac{u}{m-u}, & \mathrm{x} \in[m, u] \\ 0, & \text { otherwise, }\end{cases}
$$

where $l \leq m \leq u$. And $l, m$, and $u$ represent the lower, middle, and upper value of $M$, respectively. Table 4 shows the Fuzzified Satty's nine scale for triangular fuzzy number.

Table 4. Fuzzified Satty's Scale for Triangular Fuzzy Number[31].

\begin{tabular}{cc}
\hline Intensity Of Importance & Definition \\
\hline$(1,1,1+d)$ & Equal importance of elements \\
$(3-d, 3,3+d)$ & Moderate importance of one element relative to another \\
$(5-d, 5,5+d)$ & Strong importance of one element relative to another \\
$(7-d, 7,7+d)$ & Very strong importance of one element relative to another \\
$(9-d, 9,9)$ & Extreme importance of one element relative to another \\
$(x-d, x, x+d), x=2,4,6,8$ & Intermediate values between two adjacent judgements \\
\hline
\end{tabular}

In order to implement FAHP, the following steps are required [24]. And all the following equations for FAHP are not covered in this research.

- Step 1: Fuzzification the relative important matrix

Using the triangular fuzzy number, RM, equation (1) is reformed as follows

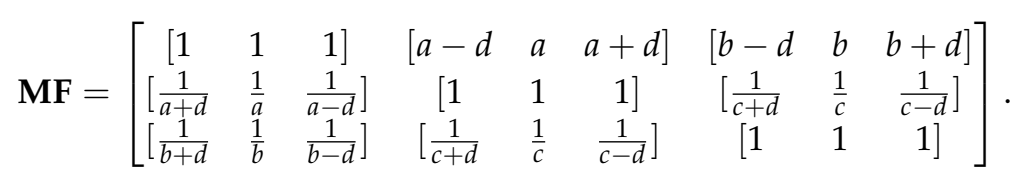

where $M F$ represents fuzzified relative importance matrix. According to equation (19), the $\mathbf{R M}_{\mathbf{1}}$ is

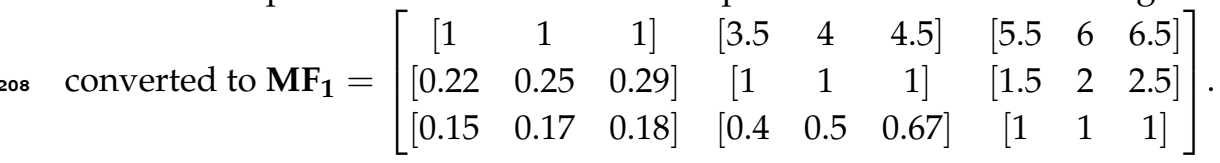

- Step 2: Calculation of fuzzy synthetic extent as follows 
210 The fuzzy synthetic extent of $M F$ is calculated as follows

$$
S_{i}=\sum_{j=1}^{m} M F_{g_{i}}^{j} \odot\left[\sum_{i=1}^{n} \sum_{j=1}^{m} M F_{g_{i}}^{j}\right]^{-1}
$$

${ }_{211}$ where $S_{i}$ is the ith synthetic extent and $M F_{g_{i}}^{j}$ is triangular fuzzy number. The operator $\odot$ is defined as

$$
\begin{gathered}
\left(l_{1}, m_{1}, u_{1}\right) \odot\left(l_{2}, m_{2}, u_{2}\right)=\left(l_{1} \times l_{2}, m_{1} \times m_{2}, u_{1} \times u_{2}\right) \\
\left(l_{1}, m_{1}, u_{1}\right)^{-1}=\left(\frac{1}{l_{1}}, \frac{1}{m_{1}}, \frac{1}{u_{1}}\right)
\end{gathered}
$$

212

213

- Step 3: Calculation of priority vectors of fuzzy AHP

Once fuzzy synthetic extent is obtained, the vectors of weights under defined objectives is derived. By the principle of the comparison of fuzzy numbers [32], the degree of possibility of $M_{2}=\left(l_{2}, m_{2}, u_{2}\right), \geq$ $M_{1}=\left(l_{1}, m_{1}, u_{1}\right)$ is defined as

$$
V\left(M_{2} \geq M_{1}\right)=\sup _{y \geq x}\left[\min \left(\mu_{M_{1}}(x), \mu_{M_{2}}(y)\right)\right]
$$

and can be equivalently expressed as

$$
\begin{aligned}
V\left(M_{2} \geq M_{1}\right)= & h g t\left(M_{1} \cap M_{2}\right)=u_{M_{1}}(d) \\
& =\left\{\begin{array}{l}
1, \text { if } m_{2} \geq m_{1} \\
0, \text { if } l_{1} \geq u_{2} \\
\frac{l_{1}-u_{2}}{\left(m_{2}-u_{2}\right)-\left(m_{1}-l_{1}\right)}, \text { otherwise, }
\end{array}\right.
\end{aligned}
$$

where $h g t$ and $d$ represent the highest intersection point and $\mathrm{x}$ coordinate of the two fuzzy number as shown in figure 9.

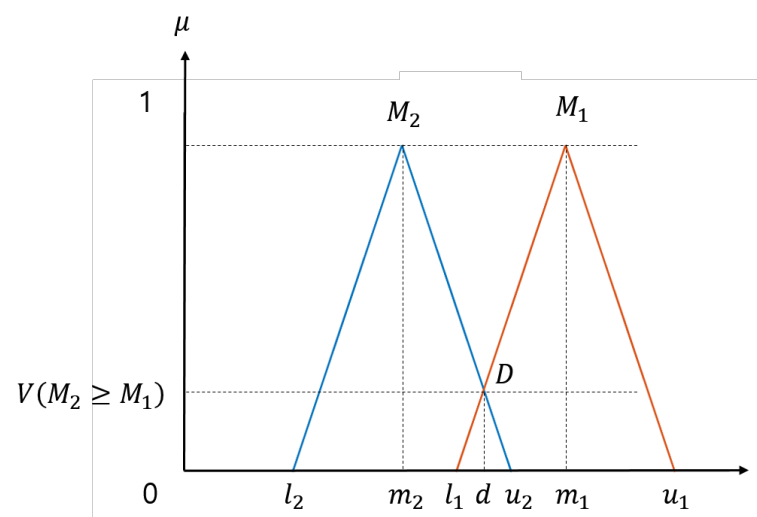

Figure 8. The intersection between $M_{1}$ and $M_{2}$.

The degree of possibility for a convex fuzzy number to be greater than $k$ convex fuzzy numbers $M_{1}(i=1,2, \cdots, k)$ can be defined by

$$
\begin{array}{r}
V\left(M \geq M_{1}, M_{2}, M_{3}, \ldots, M_{k}\right) \\
=\min V\left(M \geq M_{i}\right)
\end{array}
$$

by assuming

$$
d^{\prime}\left(A_{i}\right)=\min V\left(S_{i} \geq S_{k}\right)
$$


for $k=1,2, \cdots, n ; k \neq i$. Then the weight vector is given by

$$
W^{\prime}=\left(d^{\prime}\left(A_{1}\right), d^{\prime}\left(A_{2}\right), \ldots, d^{\prime}\left(A_{n}\right)\right)^{T}
$$

where $A_{i}(i=1,2, \cdots, n)$ are $n$ elements. By normalizing equation (27) the weight vector is given

$$
W=\left(d\left(A_{1}\right), d\left(A_{2}\right), \ldots, d\left(A_{n}\right)\right)^{T}
$$

\section{Simulation II (FAHP based path planning)}

In this Section, FAHP based mobile robot path planning is simulated on the same factory layout environment to overcome the aforementioned shortcomings of AHP. As the first step, $\mathbf{R} \mathbf{M}_{\text {dist }}$ and $\mathbf{R} \mathbf{M}_{\text {safety }}$ are converted to fuzzified relative importance matrices using equation (21). And FAHP based weight vector is calculated by following equations (22)-(30). Then the multi-objective decision making is conducted according to the equations (6)-(10). In Figure 9, FAHP based simulation results are displayed. It is shown that each of fuzzified RM plans different path.

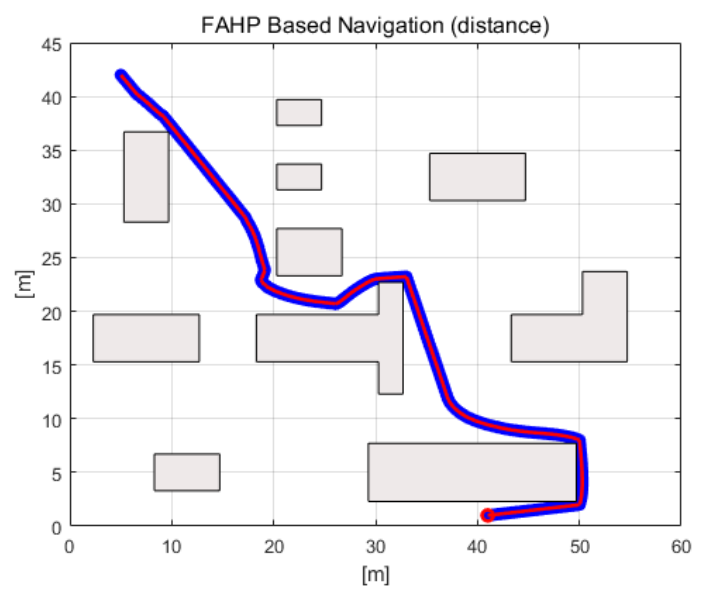

(a)

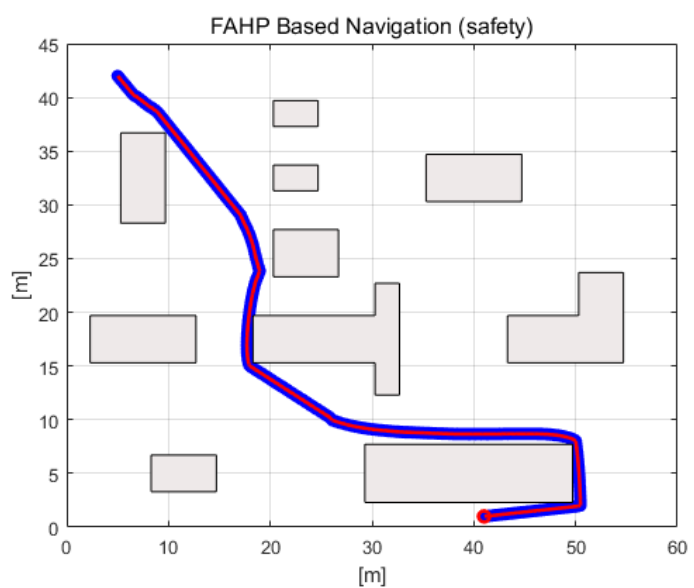

(b)

Figure 9. Safety margin under obstacles.: (a) FAHP based path planning with short distance preference (b) FAHP based path planning with high safety preference

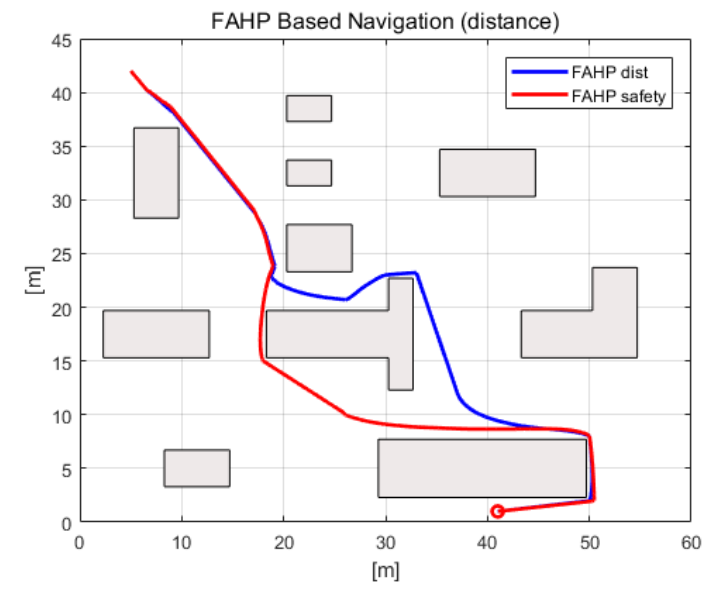

Figure 10. Comparison between short distance and high safety RM based FAHP path planning

Through the analysis of the simulation results, the travel distance of $81.01 \mathrm{~m}$, the safety score of 65.95 and 14 steep turns are found in the case of high weights for short distances. However, the safety priority strategy indicates $81.63 \mathrm{~m}, 98.11$ safety scores, and 10 steep rotation performance for each 
investigating items. The FAHP with the highest weight on distance performs shorter travel than the other preference while the highest weight on the safety achieves the higher safety score than the other. Figure 10 shows comparison path of the two path planning strategy. As with AHP, it is confirmed that FAHP based path planning follows the user's intention well.

\section{Simulation III (Artificial potential field based path planning)}

In this Section, the performances of AHP and FAHP based path planning are compared with conventional path planning strategy, artificial potential field (APF) [6]. Because the details of the APF are explained well in [6], this paper does not cover them. The basic idea of APF is generating a vector for the robot to navigate by the summation of attractive field of the goal and the repulsive field of the obstacles. Figure 11 show the simulation results with APF. And APF is composed with attractive potential, repulsive potential, and potential field as shown in Figure 11. The upper circle represents starting position and the lower circle means the target.

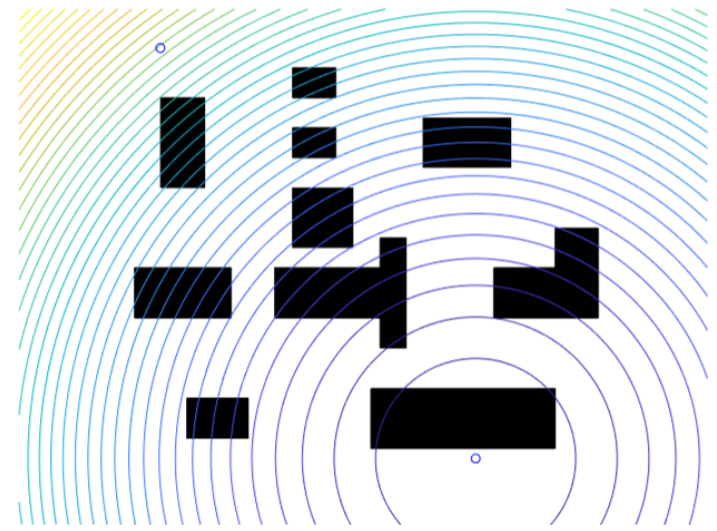

(a)

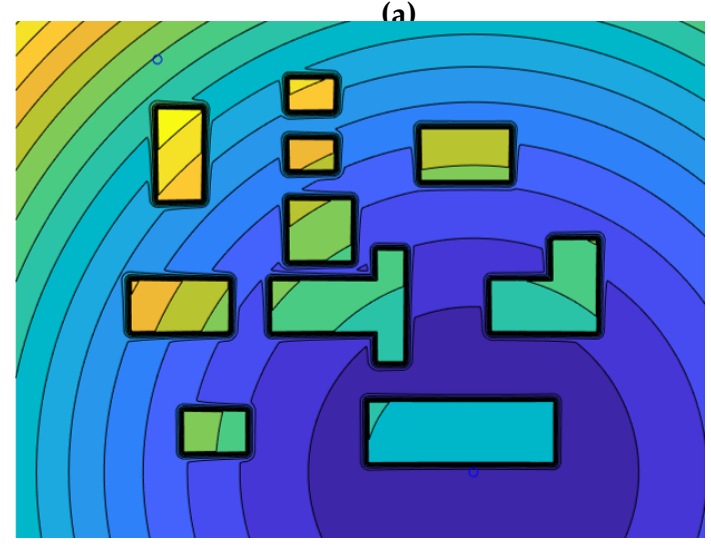

(c)

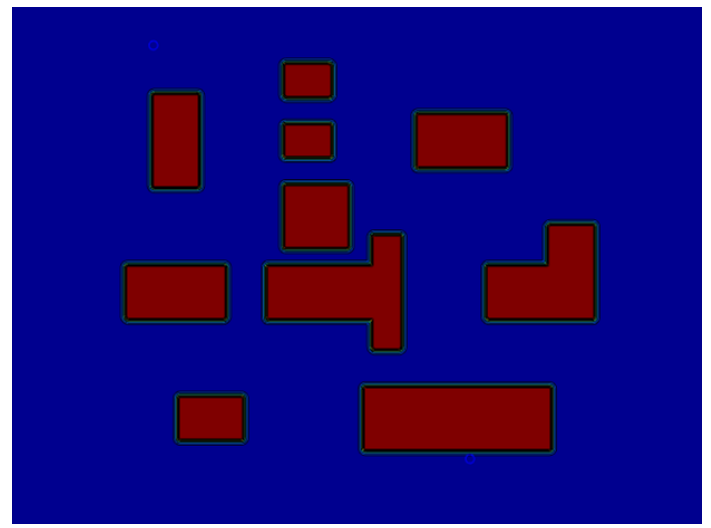

(h)

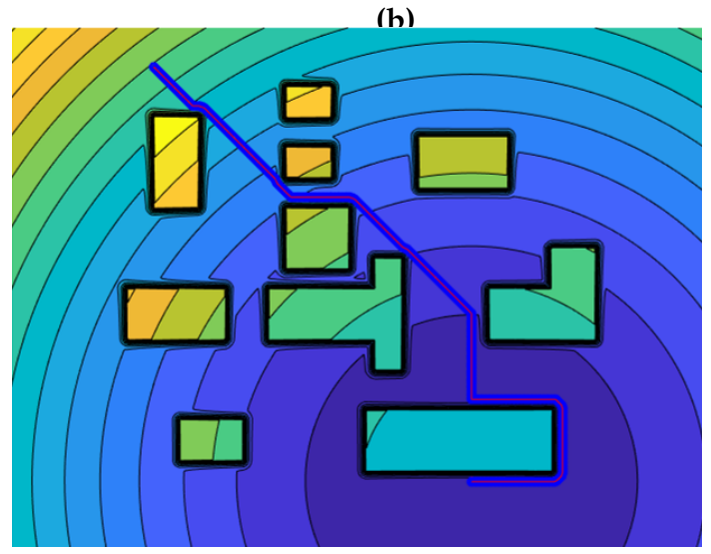

(d)

Figure 11. APF based path planning results: (a) Attractive potential (b) Repulsive potential (c) Potential field (d) APF based navigation

Table 5 indicates the performance comparison among AHP, FAHP, and APF. In most cases, AHP and FAHP are superior performance in traveling distance and the number of steep turns. When the navigation performances of AHP and FAHP are compared. FAHP dist travels shorter than $A H P_{\text {dist }}$. 
Table 5. Simulation results comparison between AHP, FAHP, and APF.

\begin{tabular}{cccc}
\hline Method & Traveling distance $[\mathrm{m}]$ & Safety score & Steep turns \\
\hline$A H P_{\text {dist }}$ & 81.46 & 50.83 & 15 \\
$A H P_{\text {safety }}$ & 84.18 & 85.30 & 12 \\
$F A H P_{\text {dist }}$ & 81.01 & 65.95 & 14 \\
$F A H P_{\text {safety }}$ & 81.63 & 98.11 & 10 \\
$A P F$ & 81.80 & N/A & 46 \\
\hline
\end{tabular}

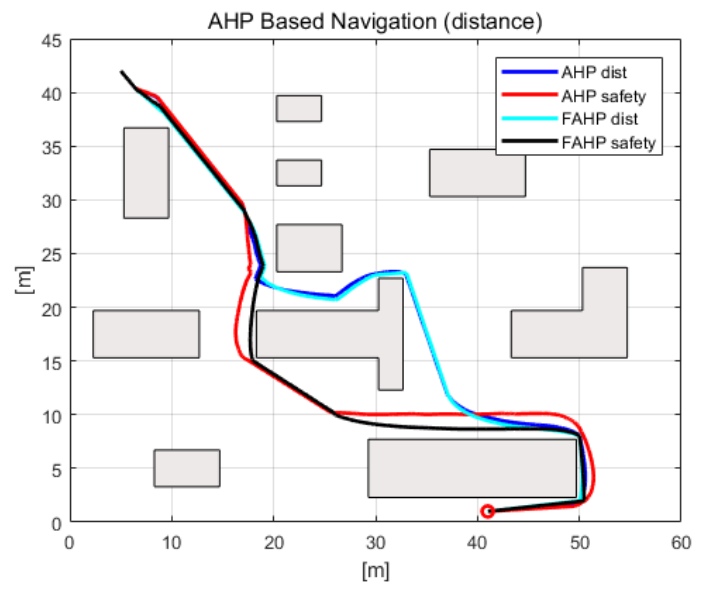

(a)

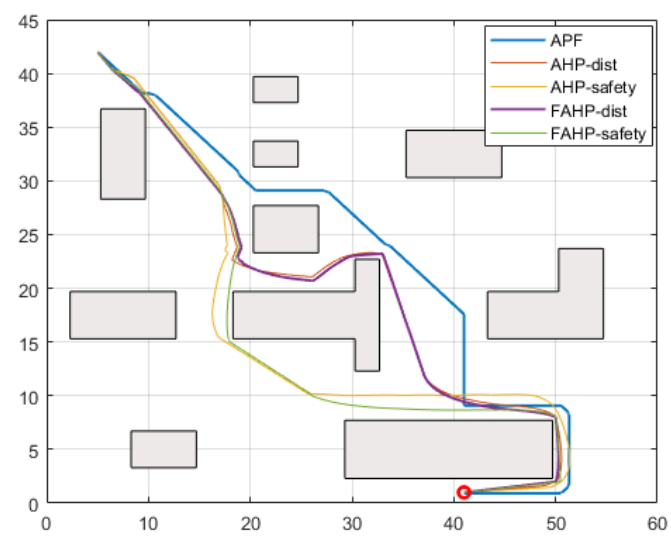

(b)

Figure 12. Navigation results.: (a) Navigation path comparison between AHP and FAHP (b) Navigation path comparison among all methods

As shown in Figure 12 (a), FAHP based decision takes more sophisticated solution than AHP. For example, Due to the AHP decision making utilizes nine-point integer scale to describe the importance between objectives it cannot handle the uncertainties, however, FAHP can decide under uncertain decision making conditions [26]. As shown in table 5, Path planning based on AHP is somewhat extreme in terms of the defined relative importance metrics. For example, distance-priority cases have lower safety performance, and safety-priority cases have lower distance performance. However, when FAHP is utilized for path planning under safety first strategy it also selects a solution that has high performance in distance reduction as shown in table 5. Among all methods, short distance travel preference based FAHP can plan the minimum travel distance path. And safety preference based FAHP plans a path that guarantees safe logistics process. The superiority of AHP-based path planning is testified by comparing the performance of mobile robot navigation using APF-based method. And it is demonstrated that FAHP compensates the weaknesses of AHP.

\section{Conclusions}

In this paper, a mobile robot path planning strategy based on multi-objective decision making framework, Analytic Hierarchy Process (AHP), has been studied. The main advantage of AHP is that decisions are made through definitions of importance among the objects being considered. However, the conventional AHP, a nine-point scale is utilized to apply decision maker's preference about relative importance between objectives. Therefore, AHP is inappropriate for making decisions under uncertain conditions. In this study, FAHP was proposed to compensate for the weakness of AHP. Through simulations, mobile robot path planning performance based on AHP was verified. The application of the FAHP method also confirmed the overall improvement in navigation performances such as distance of travel, collision safety, and rapid rotation.

Author Contributions: Conceptualization, C.K.; methodology and simulation, C.K. and Y.K.; validation, H.Y.; writing-original draft preparation, C.K.; writing-review and editing, Y.K. and H.Y. 
Conflicts of Interest: The authors declare no conflict of interest.

\section{References}

1. Siegwart, R.; Nourbakhsh, I.R.; Scaramuzza, D. Introduction to autonomous mobile robots; MIT press, 2011.

2. Raja, P.; Pugazhenthi, S. Optimal path planning of mobile robots: A review. International journal of physical sciences 2012, 7, 1314-1320.

3. Mac, T.T.; Copot, C.; Tran, D.T.; De Keyser, R. Heuristic approaches in robot path planning: A survey. Robotics and Autonomous Systems 2016, 86, 13-28.

4. Lumelsky, V.J.; Skewis, T. Incorporating range sensing in the robot navigation function. IEEE Transactions on Systems, Man, and Cybernetics 1990, 20, 1058-1069.

5. Kamon, I.; Rivlin, E.; Rimon, E. A new range-sensor based globally convergent navigation algorithm for mobile robots. Proceedings of IEEE International Conference on Robotics and Automation. IEEE, 1996, Vol. 1, pp. 429-435.

6. Latombe, J.C. Robot motion planning; Vol. 124, Springer Science \& Business Media, 2012.

7. Rimon, E.; Koditschek, D.E. Exact robot navigation using artificial potential functions. Departmental Papers (ESE) 1992, p. 323.

8. Borenstein, J.; Koren, Y. The vector field histogram-fast obstacle avoidance for mobile robots. IEEE transactions on robotics and automation 1991, 7, 278-288.

9. Ulrich, I.; Borenstein, J. VFH/sup*: Local obstacle avoidance with look-ahead verification. Proceedings 2000 ICRA. Millennium Conference. IEEE International Conference on Robotics and Automation. Symposia Proceedings (Cat. No. 00CH37065). IEEE, 2000, Vol. 3, pp. 2505-2511.

10. Susnea, I.; Minzu, V.; Vasiliu, G. Simple, real-time obstacle avoidance algorithm for mobile robots. 8th WSEAS International Conference on Computational Intelligence, Man-Machine Systems and Cybernetics (CIMMACS 2009), 2009.

11. Saska, M.; Macas, M.; Preucil, L.; Lhotska, L. Robot path planning using particle swarm optimization of Ferguson splines. 2006 IEEE Conference on Emerging Technologies and Factory Automation. IEEE, 2006, pp. 833-839.

12. Seder, M.; Petrovic, I. Dynamic window based approach to mobile robot motion control in the presence of moving obstacles. Proceedings 2007 IEEE International Conference on Robotics and Automation. IEEE, 2007, pp. 1986-1991.

13. Satty, T.L. The analytic hierarchy process; New York: McGraw-Hill New York, 1980.

14. Saaty, T.L. Decision making for leaders: the analytic hierarchy process for decisions in a complex world; RWS publications, 1990.

15. Tseng, Y.F.; Lee, T.Z. Comparing appropriate decision support of human resource practices on organizational performance with DEA/AHP model. Expert Systems with Applications 2009, 36, 6548-6558.

16. Niaraki, A.S.; Kim, K. Ontology based personalized route planning system using a multi-criteria decision making approach. Expert Systems with Applications 2009, 36, 2250-2259.

17. Chen, M.K.; Wang, S.C. The critical factors of success for information service industry in developing international market: Using analytic hierarchy process (AHP) approach. Expert Systems with Applications 2010, 37, 694-704.

18. Vidal, L.A.; Sahin, E.; Martelli, N.; Berhoune, M.; Bonan, B. Applying AHP to select drugs to be produced by anticipation in a chemotherapy compounding unit. Expert Systems with Applications 2010, 37, 1528-1534.

19. Lee, S.H. Using fuzzy AHP to develop intellectual capital evaluation model for assessing their performance contribution in a university. Expert systems with applications 2010, 37, 4941-4947.

20. Kim, C.; Langari, R. Analytical Hierarchy Process and Brain Limbic System combined strategy for mobile robot navigation. 2010 IEEE/ASME International Conference on Advanced Intelligent Mechatronics. IEEE, 2010, pp. 967-972.

21. Chen, P.Y.; Wu, J.K.; Pai, N.S.; Lai, Y.C. Design and Implementation of an Autonomous Parking Controller Using a Fuzzy controller and AHP for Car-Like Mobile Robot. International Journal of Computer, Consumer and Control 2014, 3, 27-39.

22. Kim, C.; Langari, R. Adaptive analytic hierarchy process-based decision making to enhance vehicle autonomy. IEEE Transactions on Vehicular Technology 2012, 61, 3321-3332. 
23. Van Laarhoven, P.J.; Pedrycz, W. A fuzzy extension of Saaty's priority theory. Fuzzy sets and Systems 1983, 11, 229-241.

24. Chang, D.Y. Applications of the extent analysis method on fuzzy AHP. European journal of operational research 1996, 95, 649-655.

25. Anand, M.D.; Selvaraj, T.; Kumanan, S.; Johnny, M.A. Application of multicriteria decision making for selection of robotic system using fuzzy analytic hierarchy process. International Journal of Management and Decision Making 2008, 9, 75.

26. Kilincci, O.; Onal, S.A. Fuzzy AHP approach for supplier selection in a washing machine company. Expert systems with Applications 2011, 38, 9656-9664.

27. Shin, J.; Park, Y.; Kim, T. Evaluation of inland inundation risk in urban area using fuzzy AHP. Journal of Korea Water Resources Association 2014, 47, 789-799.

28. Mosadeghi, R.; Warnken, J.; Tomlinson, R.; Mirfenderesk, H. Comparison of Fuzzy-AHP and AHP in a spatial multi-criteria decision making model for urban land-use planning. Computers, Environment and Urban Systems 2015, 49, 54-65.

29. Ravankar, A.; Ravankar, A.; Kobayashi, Y.; Emaru, T. Optimal Robot Path Selection Using Fuzzy Analytical Hierarchical Process. Multidisciplinary Digital Publishing Institute Proceedings, 2017, Vol. 2, p. 111.

30. Kabir, G.; Akhtar Hasin, M.A. Multi-criteria inventory classification through integration of fuzzy analytic hierarchy process and artificial neural network. International Journal of Industrial and Systems Engineering 2013, 14, 74-103.

31. Noorul Haq, A.; Kannan, G. Design of an integrated supplier selection and multi-echelon distribution inventory model in a built-to-order supply chain environment. International Journal of Production Research 2006, 44, 1963-1985.

32. Chang, D.Y. Extent analysis and synthetic decision. Optimization techniques and applications 1992, 1, 352-355. 